\title{
Jóvenes transitando a la educación posobligatoria: el reflejo de la injusticia hermenéutica en sus discursos ${ }^{1}$
}

\author{
Young People in Transition to Post-Compulsory Education: \\ The Reflect of Hermeneutical Injustice in their Discourses
}

\author{
Alberto Sánchez Rojo y Miriam Prieto Egido²
}

\begin{abstract}
Resumen
Los factores que influyen en las elecciones de los y las jóvenes a la hora de transitar de la etapa educativa obligatoria a la postobligatoria han sido ampliamente estudiados. La confluencia de factores estructurales y de agencia en las transiciones escolares ha sido probada. Sin embargo, estos resultados no necesariamente reflejan la conciencia que ellos y ellas tienen de dichos condicionantes, así como la medida en que consideran que estos les afectan. Sostiene Miranda Fricker (2017) que cuando una grieta en los recursos de interpretación colectivos hace que una o varias personas se encuentren en posición de desventaja con respecto a la comprensión de sus propias experiencias sociales, está teniendo lugar un caso de injusticia hermenéutica. Este trabajo, a través del análisis de 29 entrevistas desarrolladas con estudiantes de Bachillerato y Formación Profesional de cuatro centros de la ciudad de Madrid, pretende mostrar hasta qué punto los discursos juveniles en torno a sus propias transiciones son representativos de este tipo de injusticia. Los resultados muestran la existencia de diferencias estructurales en las trayectorias seguidas por el alumnado de Bachillerato y de Formación Profesional, que se concretan en su carácter uniforme o discontinuo; sin embargo, los discursos de los y las jóvenes reflejan, por un lado, una negación de todo tipo de babitus, y, por otro lado, la consideración de las elecciones y transiciones como resultado de la voluntad y/o la capacidad individual, situando la responsabilidad de las elecciones y sus consecuencias en los sujetos.
\end{abstract}

\section{Palabras clave}

Educación postobligatoria, transiciones escolares, trayectorias educativas, injusticia hermenéutica, Madrid.

\section{Abstract}

The factors influencing young people choices in their transitions from compulsory to post-compulsory education have been widely addressed. The convergence between structural factors and agency in school transitions has been proved. However, these results do not necessarily reflect their awareness of these determining factors, as well as the extent in which they consider themselves affected. Miranda Fricker (2017) claims that when a breach in the collective interpreting resources makes one or some individuals be in a disadvantageous position regarding their understandings of their own social experiences, a case of hermeneutical injustices is taking place. This paper, through the analysis of 29 interviews carried out with students of baccalaureate and intermediate vocational training belonging to four schools placed in Madrid city, aims to analyze to what extent the students' discourses about their transitions are representative of this kind of injustice. Results display the existence of structural differences in the trajectories followed by students from baccalaureate and intermediate vocational training, specified in their smooth or discontinuous character; however, young people discourses reflect, on the one hand, the denial of any babitus, and, on the other, the understanding of their own choices and transitions as the result of their will and/or individual capability, placing the responsibility of the choices and their consequences on the individuals.

\section{Keywords}

Post-compulsory education; school transitions; educational trajectories; hermeneutical injustice; Madrid.

\section{Cómo citar/Citation}

Sánche Rojo, Alberto y Prieto Egido, Miriam (2020). Jóvenes transitando a la educación posobligatoria: el reflejo de la injusticia hermenéutica en sus discursos. Revista de Sociología de la Educación-RASE, 13 (4), 579-596. http://dx.doi.org/10.7203/RASE.13.4.17843.

\footnotetext{
Este trabajo forma parte del proyecto de investigación «La construcción de oportunidades educativas post-16. Un análisis de las transiciones a la educación secundaria postobligatoria en contextos urbanos», financiado por el Ministerio de Economía, Industria y Competitividad del Gobierno de España, dentro del Programa Estatal de Fomento de la Investigación Científica y Técnica de Excelencia - Subprograma Estatal de Generación de Conocimiento (Ref. CSO-2016-80004).

2 Alberto Sánchez Rojo, Universidad Complutense de Madrid, asanchezrojo@ucm.es; Miriam Prieto Egido, Universidad Autónoma de Madrid, miriam.prieto@uam.es
} 


\section{Introducción}

La manera por la que una persona transita por el sistema educativo, optando por unas vías o por otras, no es nunca neutral; de hecho, la transición es «un procedimiento clave, tanto individual como colectivo, que no solo se refiere a un momento concreto, sino que se desarrolla a lo largo de la vida personal, social y educativa de sus protagonistas» (Azorín, 2019: 225). Así pues, dependiendo de cada persona y sus condicionantes particulares, los caminos van siendo, en mayor o menor medida, externamente marcados, aumentando o viéndose reducidas sus oportunidades educativas, sociales y/o laborales.

Estudios clásicos de la Sociología de la Educación ya mostraron hace tiempo el condicionamiento estructural de las elecciones (Bourdieu y Passeron, 1979; Bernstein, 1975); un condicionamiento que, más allá de la clase social, hoy encontramos en el género, la raza, la capacidad y una infinidad de categorías más, siendo reflejo del reconocimiento actual de la diversidad (Petriwskyj, 2014). Sea como fuere, lo cierto es que las elecciones no se hacen nunca desde el vacío y que la pertenencia a determinados grupos o contextos acaba por marcar las trayectorias de manera injusta y desigual. Ahora bien, dicha injusticia no se encuentra simplemente en el proceso causal que lleva a la persona a tomar ciertas elecciones que, analizadas en detalle, ni siquiera podrían definirse como tales, sino que también se halla en la conciencia que las personas tengan de ese condicionamiento estructural. Es precisamente este segundo tipo de injusticia de carácter epistémico y no material, el que pretendemos aquí abordar.

Afirma Miranda Fricker que la injusticia hermenéutica se produce «cuando una brecha en los recursos de interpretación colectivos sitúa a alguien en una desventaja injusta en lo relativo a la comprensión de sus experiencias sociales» (Fricker, 2017: 18). Aplicado a las transiciones escolares, la injusticia hermenéutica se traduce en el hecho de que cuando los y las jóvenes transitan por el sistema educativo, no solamente se ven abocados a ciertas vías, condicionados por contextos que los sitúan en puntos de partida de carácter claramente desigual, sino que, siendo incapaces de detectar estas circunstancias, hacen una interpretación incompleta de su experiencia académica y escolar.

El presente artículo, tomando como referencia la noción de injusticia hermenéutica, tiene como finalidad identificar los relatos de los y las jóvenes sobre sus trayectorias, transiciones y elecciones escolares, con el objetivo de identificar qué peso conceden a los factores estructurales y cuál a la agencia. Para ello, el análisis de carácter cualitativo se centra en 29 entrevistas semiestructuradas realizadas a jóvenes que estudian el primer curso de distintas modalidades de Bachillerato y varias familias de FP, pertenecientes a cuatro centros educativos de la ciudad de Madrid.

Pasamos, a continuación, en primer lugar, a exponer los presupuestos teóricos desde los que abordamos el estudio de los relatos de los y las jóvenes con relación a sus trayectorias escolares y su transición a la educación postobligatoria. En segundo lugar, detallaremos la metodología de investigación seguida. Tras esto, en tercer lugar, indicaremos los principales resultados; y, finalmente, acabaremos exponiendo unas breves conclusiones extraídas a raíz de estos.

\section{Transiciones educativas y biografías escolares: tensiones entre habitus y agencia}

El análisis de las trayectorias educativas se ha realizado desde dos tipos de enfoques (Gambetta, 1987), cuyos planteamientos se han ido reconciliando parcialmente en las últimas décadas. Un grupo de teorías sostienen la capacidad de las personas de librarse de los condicionantes externos y explican las elecciones atendiendo a la intencionalidad de estas. En este grupo podemos destacar a Boudon (1995), quien con- 
sidera que son las razones personales e individuales, y no tanto los condicionantes previos a la acción, lo que lleva a las personas a optar por unas vías u otras. Un segundo grupo de teorías consideran que las personas difícilmente pueden evitar las influencias externas, de manera que terminan por reducir las elecciones a meros efectos de dichas influencias. En este grupo encontramos a autores como Bourdieu, quien a través del concepto de 'babitus', refiere el carácter cotidiano de todo contexto que termina inevitablemente marcando las acciones y los comportamientos. Y es que, según él, «la homogeneidad objetiva de los habitus de grupo o de clase que resulta de la homogeneidad de las condiciones de existencia es lo que hace que las prácticas y las obras sean inmediatamente inteligibles y previsibles; percibidas pues como evidentes» (Bourdieu, 2007: 101) y, en parte, por tanto, como elecciones que podrían no ser consideradas como tales.

En el caso de las transiciones educativas, en ellas se concreta la compleja interrelación entre los factores estructurales que condicionan las identidades y conductas de los sujetos, y la capacidad de agencia que define al ser humano (Furlong et al., 2011). El estudio de las trayectorias educativas desde la Sociología reveló en sus inicios el influjo de factores estructurales como la clase social, el género, la etnia, etc. (Tarabini et al., 2018). Sin embargo, los diversos cambios y transformaciones sociales que se han producido durante las últimas décadas (extensión de la escolarización, flexibilización del mercado laboral, desarrollo económico y de las tecnologías, etc.) han erosionado los marcos sociales normativos en cuyo seno el estudiantado construía su identidad, generando en las personas la necesidad de construirse a sí mismas (Cuconato y Walther, 2015). En el contexto educativo y formativo, esta erosión se ha traducido en la desestandarización de las trayectorias escolares (Walther, 2006), en lo que se ha denominado como «trayectorias yo-yo», en las que los y las jóvenes siguen trayectorias menos definidas y erráticas entre la juventud y la edad adulta (Biggart y Walther, 2005). Y, si bien es verdad que la ampliación y la desestandarización paulatina de las trayectorias ha incrementado las posibilidades y alternativas a la hora de transitar por el sistema, no por ello han aumentado las oportunidades para los colectivos más vulnerables, cuyas circunstancias desiguales hoy en día les siguen marcando de manera determinante (Tarabini y Curran, 2019)

Así pues, atender al babitus es hoy todavía muy necesario, aunque no lo sea de manera exclusiva. A partir de los años 90 del siglo pasado, las dos posturas se fueron acercando, llegando a la conclusión de que, a pesar de que a la hora de explicar las transiciones del alumnado por el sistema educativo era importante analizar los condicionantes externos, también lo era atender a las características cognitivas y emocionales de las personas; es decir, sus biografías, las cuales les hacían tomar decisiones que habrían de ser consideradas no tanto racionales como pragmáticas; o, lo que es lo mismo, en consonancia con la situación y las circunstancias (Hodkinson y Sparkes, 1997). El habitus, por tanto, influye, aunque los efectos en cada persona varíen. En este sentido, cuando hablamos de educación, no nos podemos olvidar del factor institucional, que, a pesar de estar relacionado con el social, tiene una serie de procedimientos propios que merecen ser designados de manera particular. «Las normas escolares, las prácticas y las expectativas proveen materiales de clave simbólica utilizados para que [los y las jóvenes] den sentido a sus experiencias y se definan a sí mismos» (Reay, 2010: 277). Es por esta razón por la que deben ser estudiados y tenidos en cuenta, habiendo estudios que han mostrado no sólo que tienen una gran influencia, sino que ayudan también al mantenimiento del babitus social, estando ambos relacionados de manera dialéctica (Tarabini et al., 2015). 
A esto hay que añadir que la progresiva tendencia a la individualización en el ámbito de la educación ha hecho que la configuración de los sistemas educativos se base en una igualdad de oportunidades que se logra no trabajando con colectivos, sino exclusivamente con singularidades (Saura y Luengo, 2015). De esta forma, podemos afirmar que existe una tendencia a pensar que cualquier cambio en la manera de transitar de las personas dependerá fundamentalmente de intervenciones y modificaciones sobre ellas mismas y no tanto sobre sus contextos. Esta individualización influye de manera determinante en el estudiantado que, a pesar de que pueda compartir contexto y similares experiencias, tiende a concebirse como un conjunto de personas cuyas trayectorias dependen exclusivamente de su propia agencia (McDonald, 1999). En un contexto de desestandarización en el que los condicionantes y expectativas sociales parecen diluirse, el aprendizaje se convierte en una responsabilidad personal (Brunila et al., 2011). En el caso de las transiciones, la elección de una opción escolar se convierte en un derecho y un deber aparentemente libre de influjos externos, que se presenta al alumnado como una elección libre basada únicamente en intereses y competencias personales. De esta manera, la responsabilidad, tanto de la decisión como de sus consecuencias, recae en la persona (Walther, 2006). Desde esta perspectiva, el estudio de las transiciones escolares reclama el empleo de enfoques biográficos para tratar de desentrañar los procesos individuales que sigue el alumnado al transitar y desenvolverse entre los cursos de acción institucionalizados (Cuconato y Walther, 2015).

Ahora bien, aunque desde la literatura se ha constatado el influjo de ambos tipos de factores estructurales e individuales y el carácter dialéctico que caracteriza su interrelación, de ello no se deriva necesariamente que las personas sean conscientes de la existencia de ambos factores y de su influjo en las decisiones que toman. Para completar el análisis es necesario considerar las aportaciones de la perspectiva biográfica (Casal et al., 2006) que incorpora la noción de trayectoria, y permite comprender que, si bien las elecciones pueden producirse en un momento determinado, son el resultado de la historia (personal, familiar, escolar, académica) de ellos y ellas. Desde esta perspectiva, el estudio de sus relatos acerca de sus propias percepciones sobre sus transiciones escolares constituye un elemento de interés no sólo desde la consideración de los factores individuales presentes en las elecciones, sino también desde los condicionantes estructurales. Como se ha señalado anteriormente, la injusticia hermenéutica emana de las situaciones en las que los individuos carecen de los recursos necesarios para comprender sus propias experiencias; por ello, para conocer el fenómeno de las transiciones escolares se requiere no sólo conocer los procesos que siguen las personas, sino sus relatos, con el fin de identificar su propio conocimiento y conciencia de los factores estructurales que influyen en sus trayectorias y biografías escolares. Este aspecto se ha señalado mediante el término biograficidad (biographicity en inglés), y alude a la capacidad de los individuos de reflexionar sobre su propia historia de aprendizaje en relación con condicionantes externos. De acuerdo con esta noción, dicha conciencia genera una estructura biográfica de experiencias pasadas que sirve al sujeto para ordenar las experiencias futuras; de esta manera, la propia persona aumenta y al mismo tiempo restringe sus propias posibilidades de actuación en el futuro (Alheit, 2015). Siguiendo a Hodkinson (2009: 10), «la situación es asimismo interna y subjetiva. Cómo percibimos nuestra situación es parte de nuestra situación, e influye en cómo podemos actuar y actuamos».

Ante la proliferación de discursos que tienden a primar la acción sobre las personas por encima de la acción sobre los contextos a la hora de tratar de reducir desigualdades, algunos autores reclaman la necesidad de invertir el proceso. Sin modificar el contexto, no puede lograrse un aumento real de las oportunidades (Jacovkis et al., 2020). No obstante, la acción sobre el alumnado tampoco consiste meramente 
en aumentar el número de programas y vías alternativas adaptadas a ellos y ellas, como tienden a pensar los legisladores educativos (Kantasalmi y Holm, 2017), también puede consistir en aumentar los recursos personales que los y las protagonistas tienen para reconocer e interpretar sus realidades (McCollum, 2012).

\section{Acceso a la educación postobligatoria en el sistema educativo madrileño}

El presente artículo analiza relatos de experiencia de una serie de jóvenes que acaban de transitar de educación obligatoria a posobligatoria. Teniendo el sistema educativo español un carácter comprensivo durante la etapa obligatoria, esta es la primera vez que el estudiantado tiene que enfrentarse a una elección que tendrá como resultado final la obtención de una titulación diferente -Bachillerato o Ciclo de Formación Profesional de Grado Medio (CFPGM)-. Asimismo, el estudio se sitúa en el contexto del sistema educativo madrileño, condicionado por unas características políticas e institucionales particulares, especialmente en lo que refiere a la educación postobligatoria.

La Educación Secundaria Obligatoria (ESO) en el sistema educativo español se compone de cuatro cursos, que de forma ordinaria se cursan entre los 12 y los 16 años. El cuarto curso de ESO se divide en dos opciones, enseñanzas académicas, para iniciación al Bachillerato, y enseñanzas aplicadas, orientadas a la iniciación a FP, aunque ambas opciones conducen actualmente a la obtención del Título de Graduado en ESO y permiten el acceso a ambas ramas de educación postobligatoria. A su vez, existen dos alternativas a la planificación ordinaria de ESO: los Programas de Mejora del Aprendizaje y el Rendimiento (PMAR), que se dirigen al alumnado con dificultades de aprendizaje no imputables al esfuerzo y se desarrollan en $2^{\circ}$ y $3^{\circ}$ de ESO (LOMCE, 2013, art. 27); y la Formación Profesional Básica (FPB), que constituye el primer nivel de FP y se compone de dos cursos, siendo necesario tener una edad mínima de 15 años y haber cursado el primer ciclo de ESO para acceder (LOMCE, 2013, art. 41).

La Comunidad de Madrid es una de las regiones españolas con mayor segregación escolar, menor gasto público y mayor gasto privado en educación (Murillo y Martínez-Garrido, 2018), consecuencia de un proceso de reforma iniciado en el año 2003 y caracterizado por la implantación de políticas de libertad de elección de centro, autonomía escolar y rendición de cuentas (Prieto y Villamor, 2018). En lo que respecta a la educación postobligatoria, Madrid se caracteriza por la falta de oferta, sobre todo de titularidad pública, y por la desigual distribución territorial de las enseñanzas, especialmente acusada en el caso de FP (Prieto y Rujas, 2020), lo que genera que los y las jóvenes que quieren cursar enseñanzas de FP deban optar por centros que están alejados de sus lugares de residencia.

Asimismo, existen criterios diferenciados tanto en los requisitos de acceso como en los de admisión. Para los estudios de Bachillerato se requiere estar en posesión del Título de Graduado en ESO, mientras en el caso de FPGM sirven, además, el Título Profesional Básico, el de Bachillerato o un título universitario. Otras formas de acceso son: superar todas las materias de Bachillerato; superar un curso de formación para el acceso a grado medio; o superar una prueba de acceso de edad mínima de 17 años. Con respecto a la admisión, en el caso de Bachillerato se aplican los mismos criterios que en las etapas educativas precedentes (presencia de hermanos en el centro, cercanía del domicilio familiar o lugar de trabajo, renta anual familiar, discapacidad o situación de familia numerosa (Decreto 11/2019), a los que se suma la nota media del expediente académico del último curso de ESO. Por el contrario, para la admisión en CFGM se tienen en cuenta únicamente criterios académicos (Orden 2509/2013). 


\section{Metodologías}

El objetivo del artículo es analizar los relatos de los y las jóvenes sobre sus trayectorias escolares para identificar qué peso conceden a los factores estructurales y cuál a su propia capacidad de agencia. Para llevar a cabo el análisis se han realizado 29 entrevistas semiestructuradas con estudiantes del $1^{\circ}$ curso de Bachillerato y FP de cuatro centros de la ciudad de Madrid. La investigación fue realizada dentro del marco del proyecto «La construcción de oportunidades educativas post-16. Un análisis de las transiciones a la educación secundaria postobligatoria en contextos urbanos», financiado por el Ministerio de Economía, Industria y Competitividad del Gobierno de España (Ref. CSO-2016-80004), cuyo equipo elaboró y validó el contenido de las entrevistas. La elección de los y las jóvenes se hizo atendiendo a criterios de edad, género y variedad en las titulaciones y centros. En cuanto al contenido, se realizaron preguntas acerca de cuatro ámbitos: trayectoria educativa, proceso de transición a educación postobligatoria, primeras experiencias en educación postobligatoria y familia y condiciones socioeconómicas. Para la selección de los centros se combinaron tres criterios: que impartiesen la etapa de ESO y que además dispusiesen de las dos ramas de educación postobligatoria, la titularidad (pública y concertada) y la composición social ${ }^{3}$. Con respecto al primer criterio, las modalidades de Bachillerato incluidas en los centros seleccionados fueron Ciencias y Humanidades y Ciencias Sociales, así como también Bachillerato Internacional, mientras los ciclos de FP representados en la muestra fueron Gestión, Auxiliar de Enfermería, Auxiliar de Farmacia, Atención a la Dependencia, Informática y Peluquería. Con respecto a la titularidad y composición social de los centros, contamos con un centro público y uno concertado en contextos de nivel socioeconómico bajo, un centro público en nivel socioeconómico medio y uno concertado en nivel socioeconómico alto.

Las entrevistas con los y las jóvenes se llevaron a cabo durante el curso escolar 2018/2019. El análisis de estas reveló la emergencia inductiva de las categorías «voluntad» y «capacidad» y «negación de factores estructurales» (especialmente género y nivel socioeconómico). La categoría «voluntad» se aplicó en aquellos casos en los que los y las jóvenes aludían a cuestiones subjetivas (deseos, intereses, preferencias, etc.) como factores causantes de las elecciones académicas, mientras «capacidad» se empleó en los casos en los que se referían a aspectos relativos al rendimiento, talentos, aptitudes, inteligencia, etc. «Negación de factores estructurales» se aplicó en los casos en los que las personas entrevistadas negaban la influencia de factores externos y ajenos a su voluntad. Para el análisis de las trayectorias se ha aplicado de forma deductiva la clasificación de Walther et al. (2015), concretamente las siguientes categorías: académica uniforme, académica discontinua, profesional uniforme y profesional discontinua. La categoría académica se aplica en los casos de estudiantes que cursan Bachillerato, y la profesional se aplica a los estudiantes que cursan FP. La trayectoria se categoriza como continua cuando transcurre de acuerdo con los cursos y las edades establecidos de manera ordinaria en la ordenación del sistema educativo español, y discontinua cuando se produce alguna repetición o abandono.

\footnotetext{
3 Si bien estos fueron los criterios seleccionados inicialmente, dado que la muestra de centros que cumplía los tres requisitos era limitada y que la participación de los centros en la investigación queda a su voluntad, solo se pudieron llevar a cabo entrevistas en tres centros. En el transcurso de los contactos un cuarto centro se ofreció a participar en las entrevistas, y si bien encajaba en las necesidades de la muestra en los criterios de titularidad y composición social, carecía de FP. Ante el hecho de que los centros en los que se habían realizado las entrevistas ofertaban distintas familias de FP y por lo tanto el número de estudiantes de FP a los que se había entrevistado era más elevado, se decidió incluir en la investigación a ese centro para aumentar la muestra de estudiantes de Bachillerato.
} 


\section{Resultados}

\subsection{Trayectorias escolares: linealidad en Bachillerato versus discontinuidad en FP}

La transición de los y las jóvenes que estudian Bachillerato coinciden con lo que Walther et al. (2015) denominan trayectorias «académicas uniformes» (ver tabla I); es decir, trayectorias sin rupturas. Esto encaja con cierta continuidad naturalizada por la estructura y organización del sistema educativo español, en el que existe una clara continuidad entre el enfoque académico de ESO y Bachillerato (Tarabini y Jacovkis, 2019).

Tabla I. Trayectorias de los y las jóvenes que estudian Bachillerato ${ }^{4}$

\begin{tabular}{|c|c|c|c|}
\hline JOVEN & SÍNTESIS TRAYECTORIA & REPETICIONES & CLASIFICACIÓN \\
\hline C_B_IB_chico & $\begin{array}{l}\text { Cursó los estudios previos a Bachillerato en México. Está cursando } 1^{\circ} \text { de Bachillerato Internacional y en el futuro quiere estudiar una } \\
\text { carrera relacionada con ingeniería. }\end{array}$ & 0 repeticiones & Académica uniforme \\
\hline C_B_BB_chica & $\begin{array}{l}\text { Cursó los estudios previos a Bachillerato en el mismo centro (a excepción de un curso que lo hizo en Reino Unido). Irá a la } \\
\text { universidad. . }\end{array}$ & 0 repeticiones & Académica uniforme \\
\hline P_B_Ccs_chica & Cursó ESO en el mismo centro en el que estudia Bachillerato. Irá a derecho, quiere ser policía y entrar como inspectora. & 0 repeticiones & Académica uniforme \\
\hline P_B_Ccs_chico & $\begin{array}{l}\text { Repitió } 4^{0} \text { de ESO y este curso está repitiendo } 1^{0} \text { de Bachillerato. Quiere ir a la universidad y estudiar programación. Se planteó } \\
\text { estudiar FP, pero sus padres le hicieron ver que tiene mejor reputación Bachillerato. }\end{array}$ & 2 repeticiones & Académica discontinua \\
\hline P_B_CCSS_chica & $\begin{array}{l}\text { Cursó ESO en el mismo centro en el que estudia Bachillerato. Repitió } 3^{\circ} \text { de ESO. Quiere estudiar Magisterio. Nunca se ha planteado } \\
\text { estudiar FP porque sus padres consideran mejor Bachillerato. }\end{array}$ & 1 repetición & Académica discontinua \\
\hline P_B_CCSS_chico & $\begin{array}{l}\text { Cursó hasta ESO en un concertado; repitió } 3^{\circ} \text { de ESO en modalidad PMAR. Quiere ser policía, dependiendo de la calificación que } \\
\text { obtenga en EVAU se planteará ir a la universidad. No se plantea estudiar FP. }\end{array}$ & 1 repetición & Académica uniforme \\
\hline C_B_Ccs_chical & Se incorporó al Sistema educativo español en $1^{0}$ de Bachillerato. Quiere estudiar ingeniería y ser jefa. & 0 repeticiones & Académica uniforme \\
\hline C_B_Ccs_chicol & $\begin{array}{l}\text { Cursó ESO con normalidad. Eligió los estudios postobligatorios en función de su experiencia en ESO. Siempre ha tenido claro que } \\
\text { quería estudiar Bachillerato, por que tiene más salidas. No sabe lo que hará cuando termine Bachillerato }\end{array}$ & 0 repeticiones & Académica uniforme \\
\hline C_B_Ccs_chico2 & Cursó ESO con normalidad. Quiere estudiar veterinaria. & 0 repeticiones & Académica uniforme \\
\hline C_B_Ccs_chica2 & $\begin{array}{l}\text { Cursó ESO con normalidad. Quiere ir a la universidad, pero no sabe qué carrera estudiar. No descarta FP si en Bachillerato no le va } \\
\text { bien. }\end{array}$ & 0 repeticiones & Académica uniforme \\
\hline C_B_CCSS_chica & Cursó ESO con normalidad. Quiere estudiar Comunicación Audiovisual. & 0 repeticiones & Académica uniforme \\
\hline C_B_CCSS_chico & Cursó ESO con normalidad, si bien en $4^{0}$ de ESO experimentó muchas dificultades académicas. Quiere estudiar Periodismo. & 0 repeticiones & Académica uniforme \\
\hline
\end{tabular}

Fuente: elaboración propia.

Solo tres estudiantes reportan un cierto desvío con respecto a las trayectorias seguidas por los jóvenes que cursan Bachillerato. Dos de los estudiantes entrevistados han repetido un curso y un tercero ha repetido dos veces. Además, en el caso de uno de los estudiantes que han repetido $3^{\circ}$, el segundo año lo cursa siguiendo PMAR. Estos desvíos no provocan que dichas trayectorias dejen de considerarse lineales (dado que las repeticiones están comprendidas dentro de la regulación del sistema educativo y pueden, por tanto, considerarse parte de él). Hablaríamos en este caso de trayectorias académicas discontinuas, siguiendo la clasificación de Walther et al., (2015), dado que las trayectorias escolares conducen igualmente a la educación secundaria postobligatoria en la rama académica, si bien reflejan un desarrollo más heterogéneo debido a bajo rendimiento.

Estos casos parecen representar una excepción a la norma de que el alumnado que opta por la rama de Bachillerato responde a un perfil académico de éxito. No obstante, si profundizamos en los relatos de los y las jóvenes, encontramos que existen atribuciones, propias o de las familias, acerca de la FP,

4 Los datos relativos a los y las jóvenes se han anonimizado mediante códigos que reflejan la titularidad del centro (C para concertados y P para públicos), la opción de educación postobligatoria que cursan (B para Bachillerato), la modalidad (IB para Bachillerato Internacional, Ccs para Ciencias y CCSS para Humanidades y Ciencias sociales) y el sexo (chico o chica). 
que les generan una barrera cognitiva que impide que opten por esa opción, ya que la vinculan con un descenso en la escala social y con el fracaso académico (Horcas y Giménez, 2017):

\begin{abstract}
«Es que, aunque me cueste reconocerlo, si tú dices: "He hecho un grado medio, un grado superiory he ido a la carrera a «he hecho Bachillerato y he ido a la carrera", te miran de una forma distinta, entonces yo lo be visto y lo comprendo». (P_B_Ccs_chico).
\end{abstract}

«No, o sea, pero porque mis padres siempre han tenido carrera, o sea, tienen... han opositado y todo entonces tienen Bachillerato, pero si les dices lo de FP, pues es como... "mejor vete a Bachillerato y...”》. (P_B_CCSS_chica).

Por el contrario, los relatos de los y las jóvenes que estudian FP revelan mayor variabilidad en las trayectorias escolares (ver tabla II). La linealidad de las trayectorias de los jóvenes que estudian Bachillerato contrasta con el carácter errático, de salida y entrada en el sistema educativo, de las trayectorias escolares de los y las jóvenes que estudian FP. Las transiciones no-lineales «implican pausas, rupturas, cambios de dirección» (Furlong et al., 2006: 231) con respecto a las trayectorias marcadas por la estructura y organización del sistema educativo español. De acuerdo con Walther et al., (2015), las trayectorias profesionales pueden clasificarse en uniformes, englobándose bajo esta denominación aquellas trayectorias que conducen a la formación profesional sin rupturas en el camino marcado por la organización del sistema educativo, ya sea por deseo propio de los y las jóvenes o tras un proceso de ajuste de las aspiraciones debido a un desempeño bajo en la etapa obligatoria de educación secundaria; y discontinuas, en las que el acceso a la formación profesional se produce tras rupturas en la carrera escolar, debido a distintos factores, entre ellos el fracaso académico o bajo rendimiento. En el caso de las trayectorias discontinuas o, en términos de Furlong et al., no-lineales, «pueden implicar periodos largos o repetidos de experiencias de desempleo, movimientos entre el trabajo y vueltas al sistema educativo» (2006: 231).

Así, como se puede comprobar en la tabla II, las trayectorias no-lineales emergen en los relatos de los y las jóvenes que estudian FP, especialmente con relación a experiencias de abandono del sistema educativo, en varios casos sin haber obtenido el título de Graduado en ESO, seguidos de periodos relativamente largos de empleabilidad, para posteriormente regresar al sistema educativo en la modalidad de FP. Además de la emergencia de transiciones no-lineales, en los relatos del estudiantado que está cursando FP encontramos también mayor diversidad con relación al carácter uniforme o discontinuo de las trayectorias desde la perspectiva de las opciones que ofrece el sistema educativo, ya que, como apuntamos más arriba, el acceso a FP no se hace solo a través del Título de Graduado en ESO, sino que se emplean vías alternativas como la certificación de Bachillerato, el Título de Bachillerato, FPB o las pruebas de acceso, lo que refleja que los y las jóvenes entrevistados transitan dentro del sistema educativo, siendo sus trayectorias no-lineales no solo por abandono del sistema, sino también dentro del mismo. 
Tabla II. Trayectorias de los y las jóvenes que estudian $\mathrm{FP}^{5}$

\begin{tabular}{|c|c|c|c|}
\hline JOVEN & SÍNTESIS & REPETICIONES & CLASIFICACIÓN \\
\hline P_FP_informática_chical & $\begin{array}{l}\text { Repitió curso en } 2^{\circ} \text { de Educación Primaria (EP) y } 2^{\circ} \text { de ESO. Comenzó a cursar Bachillerato, pero no le fue bien y cambió a } \\
\text { FP. Se plantea continuar con el superior. No tiene expectativas de ir ala universidad. }\end{array}$ & 2 repeticiones & Profesional discontinua \\
\hline P_FP_informática_chicol & $\begin{array}{l}\text { Se incorporó al sistema educativo español con } 14 \text { años, terminó ESO y abandonó el sistema educativo para trabajar. Tiene } \\
\text { aspiraciones de llegar a la universidad tras cursar CFPGS. }\end{array}$ & 2 repeticiones & Profesional discontinua \\
\hline P_FP_informática_chica2 & $\begin{array}{l}\text { Cursó FP Básica, superó el } 1^{0} \text { curso, pero cumplió } 18 \text { años y no pudo continuar. Abandonó los estudios. Obtuvo el título de } \\
\text { Graduado en ESO por educación de adultos. }\end{array}$ & 2 repeticiones & Profesional discontinua \\
\hline P_FP_informática_chico2 & $\begin{array}{l}\text { Se incorporó al sistema educativo español en } 1^{0} \text { de EP. Repitió } 1^{0} \text { por desconocimiento del idioma. Cursó ESO con normalidad. } \\
\text { Está cursando un CFPGM y cuando termine quiere continuar con el CFPGS. }\end{array}$ & 1 repetición. & Profesional uniforme \\
\hline P_FP_química_chico & $\begin{array}{l}\text { Repitió } 2^{0} \text { de EP. Cursó ESO con normalidad y con buenas calificaciones. Está cursando un CFPGM y quiere continuar con el } \\
\text { CFGS. No tiene expectativas de ir a la universidad. }\end{array}$ & 1 repetición & Profesional uniforme \\
\hline P_FP_química_chico & $\begin{array}{l}\text { Cursó ESO con normalidad. A continuación empezó Bachillerato, pero el primer año lo dejó para cambiarse a un CFPGM. } \\
\text { Cuando termine quiere trabajar y hacer un CFPGS. No tiene expectativas de ir a la universidad. }\end{array}$ & 0 repeticiones & Profesional continua \\
\hline C_FP_peluquería_chical & $\begin{array}{l}\text { Cursó hasta } 3^{\circ} \text { de ESO en su país de origen (repitió } 6^{\circ} \text { de EP); obtuvo el Graduado en ESO en el sistema educativo español } \\
\text { a través de educación de adultos. Cursó un (FPGM en Hostelería, y actualmente está cursando CFPGM en Peluquería (quería } \\
\text { hacer un (FPGS pero no se lo podía permitir). Quiere hacer el CFPGS y después ir a la universidad. }\end{array}$ & 1 repetición & Profesional uniforme \\
\hline C_FP_peluquería_chica2 & Repitió $3^{0}$ de ESO. Después de este CFPGM quiere estudiar otro (Estética) y después un CFPGS. & 1 repetición & Profesional uniforme \\
\hline P_FP_at.dep_chica & $\begin{array}{l}\text { Repitió } 2^{0} \text { y } 3^{\circ} \text { de ESO; en } 3^{0} \text { de ESO realizó la prueba de acceso a FP y aprobó, aunque aún así finalizó el curso de ESO. } \\
\text { Quiere estudiar el CFPGS. Si se ve capacitada le gustaría estudiar Psicología. }\end{array}$ & 2 repeticiones & Profesional discontinua \\
\hline P_FP_aux.farmacia_chica & $\begin{array}{l}\text { Repitió } 2^{\circ} \text { y } 4^{0} \text { de ESO e inició } 1^{0} \text { de Bachillerato porque quería estudiar Derecho. Abandonó los estudios y trabajó durante } \\
\text { un año. }\end{array}$ & 2 repeticiones & Profesional discontinua \\
\hline P_FP_aux.farmacia_chico & $\begin{array}{l}\text { Cursó ESO con normalidad y después continuó con Bachillerato. Al finalizar, abandonó el sistema educativo para trabajar } \\
\text { porque no le gustaba ninguna alternativa. Intentó hacer un (FPGM (Auxiliar de Enfermería) combinándolo con trabajo, pero lo } \\
\text { abandonó. Este curso ha accedido nuevamente a CFPGM. }\end{array}$ & 0 repeticiones & Profesional discontinua \\
\hline C_FP_gestión_chica & $\begin{array}{l}\text { Se incorporó en } 3^{0} \text { de ESO al sistema educativo español (en su país de origen repitió } 6^{\circ} \text { de EP). Terminó ESO por la } \\
\text { modalidad de diversificación; después empezó Bachillerato, pero cursó sólo el primer trimestre, suspendió todas y abandonó } \\
\text { los estudios por embarazo y para trabajar. }\end{array}$ & 1 repetición. & Profesional discontinua \\
\hline P_FP_gestión_chica & $\begin{array}{l}\text { Repitió } 4^{0} \text { de EP. Cursó ESO con dificultad, pero sin repetir. Quería estudiar Bachillerato para estudiar Veterinaria, pero su } \\
\text { familia y sus profesores le quitaron la idea. Miró salidas y eligió este CFPGM por descarte. }\end{array}$ & 1 repetición & Profesional uniforme \\
\hline P_FP_gestión_chico & $\begin{array}{l}\text { Cursó hasta } 4^{0} \text { de ESO con normalidad; en } 4^{\circ} \text { debía repetir, pero en lugar de eso se presentó a la prueba de acceso a CFPGM. } \\
\text { No se plantea estudiar CFPGS, en todo caso otro CFPGM. }\end{array}$ & 0 repeticiones & Profesional discontinua \\
\hline C_FP_gestión_chica & $\begin{array}{l}\text { Se incorporó al sistema educativo español con } 14 \text { años. Repitió } 2^{\circ} \text { de ESO, en } 3^{\circ} \text { de ESO tuvo muchas dificultades y comenzó } \\
\text { FPB. Accedió a CFPGM desde FPB; no se plantea estudios posteriores. }\end{array}$ & 1 repetición & Profesional uniforme \\
\hline C_FP_aux.enfermería_chico & $\begin{array}{l}\text { Experimentó dificultades al cursar ESO, repitió dos veces y pasó a una Programa de Cualificación Profesional Inicial (PCPI). } \\
\text { Al finalizar abandonó el sistema educativo y trabajó durante dos años de camarero. Accedió a CFPGM por título de ESO. No } \\
\text { cierra la puerta a cursar un CFPCS, pero sí a la universidad. }\end{array}$ & 2 repeticiones & Profesional discontinua \\
\hline C_FP_aux.enfermería_chica & $\begin{array}{l}\text { Cursó ESO con normalidad. Quería estudiar el CFPGM de Auxiliar de Enfermería, pero no obtuvo plaza, por lo que cursó un } \\
\text { año de Peluquería. Al año siguiente obtuvo plaza en Auxiliar de Enfermería. Quiere hacer un CFPGS y después la carrera de } \\
\text { Enfermería. }\end{array}$ & 0 repeticiones & Profesional uniforme \\
\hline
\end{tabular}

Fuente: elaboración propia.

Un aspecto a destacar es que, al contrario de lo que ocurre con los y las jóvenes que estudian Bachillerato, en el caso de quienes estudian FP existen dos denominadores comunes con respecto a la experiencia escolar pasada y las expectativas futuras: la mayoría ha experimentado al menos una repetición de curso, y en varios casos dos, siendo aquí los que no han repetido ningún curso la excepción. Asimismo, son también una minoría quienes manifiestan expectativas de acceso a la universidad, siendo más frecuentes las aspiraciones a CFPGS. De ello se puede concluir que, en el caso de los y las jóvenes entrevistados, la elección de FP se asocia con experiencias académicas de bajo rendimiento, y esta elección sirve también para modular las aspiraciones y expectativas futuras, que decaen con respecto al acceso a la universidad. Estos resultados coinciden con los reportados por Jacovkis et al., (2020) para el caso de la ciudad de Barcelona, en el que los y las jóvenes que estudian FP reportan

5 Para anonimizar e identificar a los y las jóvenes que estudian FP se ha empleado la misma clasificación relativa a la titularidad del centro que la empleada en Bachillerato, a continuación, se señala la opción de postobligatoria (FP) seguida del ciclo y el sexo. 
expectativas y aspiraciones de acceso a la universidad mucho menores que las de los y las jóvenes que estudian Bachillerato.

\subsection{Factores individuales como explicación de las transiciones escolares}

De acuerdo con Bourdieu y Passeron (1979) el individualismo en el que se sustenta el capitalismo hace que tanto el éxito como el fracaso tiendan a internalizarse como propios, dejando de lado la configuración misma del sistema como posible causa de uno u otro. Siguiendo esta línea, en ninguna de las entrevistas realizadas se encuentra discurso alguno que haga referencia directa a ningún aspecto del habitus como motivo del éxito o del fracaso, sino que todas las razones apuntan a cuestiones personales, dentro de las cuales destacan principalmente dos: la voluntad y la capacidad . Si bien ya existen propuestas de medidas educativas que podrían ayudar a repartir equitativamente esta responsabilidad de los resultados (González y Bernárdez-Gómez, 2019), tal y como hemos indicado, la lógica misma del sistema educativo español dificulta su implementación.

Por un lado, están quienes señalan las ganas y la voluntad que uno tenga como garantía total de éxito, pues, como afirma uno de los entrevistados,

«en general todo el mundo es capaz de hacer todo siempre y cuando se plantee de verdad bacerlo. Yo be conocido a gente que nunca quería hacer nada, pero se han puesto en serio a hacer una cosa y han logrado en unas semanas lo que otra gente se tardaba un año; entonces, la motivación y las ganas del alumno [son lo fundamental]》. (P_B_Ccs_chico).

Para eso es importante sentir interés por los estudios que se están cursando. Así, C_B_Ccs_chica2, afirma que «si te pones yo creo que todo el mundo vale para todo, pero sí es verdad que te van a salir mejor cosas que te gusten»; lo cual puede completarse con el discurso de C_B_IB_chico, quien sostiene lo siguiente: «si tú crees, o querías estudiar en principio esto, y luego has cambiado porque te gusta más esto, tú sabes las consecuencias de cambiarte, y lo que quieres. No hay mucho problema ahí, yo diría» (C_B_IB_chico). Tal y como podemos observar, se trata de descubrir los propios intereses y luchar por desarrollarse en ellos.

Por otro lado, en los discursos de los y las jóvenes encontramos también referencias a la capacidad como causa principal del éxito y el fracaso. Hemos de destacar que, si bien hay más jóvenes de Bachillerato que de FP que descargan la garantía de éxito en la voluntad, la relación se invierte cuando se trata de poner por encima la capacidad. La conclusión de Gale y Parker (2018) según la cual, para los grupos de élite la posibilidad viene regida por el deseo, mientras que para quienes pertenecen a grupos marginados, son las posibilidades las que marcan el deseo, aparece reflejada también en los relatos de los y las jóvenes entrevistados, adquiriendo aquí el término «élite» dos sentidos. Por una parte, un sentido social, pues aquellos con más recursos expresan claramente que sus posibilidades no dependen solo de los resultados, como una de las jóvenes que cursa Bachillerato (C_B_IB_chica), quien afirma que, si no le llega la nota para una universidad pública, tiene claro que irá a una universidad privada; y, por otra parte, en un sentido académico, ya que quienes están en Bachillerato inciden constantemente en sus posibilidades, como uno de los jóvenes, que afirma cursar Bachillerato de Ciencias porque «al final podré optar a más cosas» (P_B_Ccs_chico), u otro que, aunque inicialmente menciona la capacidad, luego le resta importancia, indicando que «a lo mejor [algunos] no están capacitados para meterse 
en un Bachillerato pero... no tiene por qué, si tu al final has elegido irte por Bachillerato es porque te gusta estudiar, pues lo que estás estudiando y si te vas por una FP, es que te quieres especializar« (C_B_Ccs_chico1).

Esto contrasta con experiencias como la de una de las jóvenes que cursa FP, quien teniendo una trayectoria no lineal, con dos repeticiones y habiendo accedido al CFPGM a través de prueba de acceso, no deja de repetir que los caminos por los que ha transitado no tienen que ver tanto con sus deseos sino con su capacidad, hasta el punto de decir que «me molaría, si me veo capacitada, me molaría hacer la prueba de acceso a la universidad para hacer la carrera de Psicología, pero eso ya no sé, se podría intentan» (P_FP_at.dep_chica). Todo dependerá de los resultados que vaya teniendo, que serán los que le vayan reafirmando o no su capacidad. Del mismo modo se manifiesta un joven que estudia Informática, con una trayectoria también errática, afirmando que el éxito no depende «del tipo de estudio, sino del tipo de estudiante que es cada uno; de qué eres capaz» (P_FP_informática_chico2), lo cual irá asimismo reflejado en los buenos o malos resultados. Y es que, mientras que la mayoría de los y las jóvenes entrevistados que cursan Bachillerato no relacionan los resultados con sus posibilidades, pues al final depende de las ganas que le pongan, de sus intereses y de su voluntad, muchos de los entrevistados que cursan FP, sobre todo quienes han seguido trayectorias más erráticas, equiparan sus resultados a su capacidad. Es decir, no a lo que quieren, sino a lo que pueden; no a lo que hacen, sino a lo que son.

De cualquier modo, todo el alumnado entrevistado explica sus trayectorias basándose exclusivamente en sí mismo y eximiendo de toda responsabilidad a cualesquiera agentes externos. Los resultados académicos son para los y las jóvenes una fuente de información clave para llevar a cabo sus elecciones y su logro parece depender solo de ellos, cuando en el fondo les influyen bastantes condicionantes externos (Elias y Daza, 2019). Aunque sean capaces de observar las características estructurales que les rodean, e incluso lleguen a observar ciertas consecuencias que puedan tener sobre ellos, a la hora de relatar su trayectoria, esto parece serles ajeno. La primacía del yo tiene como reverso una clara negación del peso que tienen los distintos habitus en los que se mueven.

\subsection{Negación de factores estructurales como explicación de las transiciones esco- lares}

Entre los factores estructurales referidos frecuentemente por las personas entrevistadas, aunque no considerados como influyentes en sus elecciones y trayectorias, encontramos principalmente el género, la clase social y la institución escolar. Con relación al género, afirman que no constituye un condicionante a la hora de elegir qué estudios cursar, ya sea Bachillerato o FP, o una modalidad o familia dentro de cada opción. Esta negación de los factores estructurales con relación al género se da de manera indistinta tanto entre el estudiantado de FP como de Bachillerato. En el caso de FP, esta negación se ejemplifica en el siguiente testimonio de una de las jóvenes entrevistadas: «es que también yo me asombré cuando vine aquí al Grado, porque solamente me encontré con una compañera, y digo yo: 'Somos las dos únicas chicas en clase’» (P_FP_informática_chica1). En el caso de Bachillerato, se refleja en el siguiente análisis: 
«-En mi clase somos 23 de los cuales solo 7 somos chicas. Mientras que en el $A$ que es el de Letras solo bay como 4 chicos y son veinte y no sé cuantos, o treinta y algo, no sé.

- ¿Y cuál crees tú que es la razón?

- Abi si que no lo sé. Las ingenierías igual están más llenas de chicos que de chicas. Siem-

pre ha sido así, no sé por qué. La verdad es que no... Yo no me explico por qué una chica se va a orientar más a las Letras, eso ya son cosas de cada quien».(C_B_CCSS_chica).

De la misma manera que niegan el influjo de factores estructurales de género y reducen las elecciones a cuestiones subjetivas e individuales, lo mismo sucede con los factores relativos al nivel socioeconómico. Esta negación queda reflejada en el discurso de un estudiante de FP:

$\ll-$ ¿Por qué escogiste este centro?

- Pues, diría yo que por cercanía. Porque los otros estaban muy lejos. Había que hacer transbordos en metro, asi que paso. Y cogí este.

- ¿Miraste varios sitios?

- Sí, miré varios.

- ¿Cuáles?

- Moratalazy algunos centros privados. Pero los precios están súper inflados. Los precios están muy altos. Vi uno que ponía 5000 y algo. Un año.

- Y al final este lo cogiste por la cercanía. ¿Algún motivo más?

- Creo que ninguno más, por cercanía simplemente». (P_FP_informática_chico2).

Si bien el propio joven reconoce el influjo del factor económico en su relato, dado que le llevó a descartar los centros de titularidad privada, no incluye el factor económico como un criterio o condicionante a la hora de escoger centro para estudiar FP, limitándose a la cercanía al lugar de residencia. En una línea similar transcurre el discurso de una estudiante de Bachillerato en la modalidad IB, la cual al hablar de otros compañeros que estudiaron con ella ESO en el mismo centro, y que en Bachillerato no están cursando la modalidad IB, afirma: «aparte de que cuesta más dinero, o sea, tampoco es el dinero. También porque cada uno elige cómo quiere que sea su futuro, ¿no? O sea, pues yo quiero tener una dotación internacional pues la elijo» (C_B_IB_chica).

Esto también puede encontrarse en lo referente a ciertas prácticas escolares o habitus institucional (Tarabini et al., 2015). Es el caso de uno de los jóvenes que estudia FP quien, tras relatar que en su centro se realizaba división de grupo por niveles y que él estaba en el nivel más bajo, y ante la pregunta de si esto había influido posteriormente en su trayectoria responde que «sí, porque se aprende menos» (P_FP_gestión_chico,). No obstante, un poco más adelante, al ser cuestionado sobre si alguna vez había escuchado decir que hay alumnos más válidos para unas vías que para otras, responde que «bueno, no exactamente, pero si no puedes, no puedes...», para terminar afirmando que el hecho de tomar la opción de FP o de Bachillerato «depende de la persona»; es decir, no reconociendo influencia alguna 
de las prácticas institucionales, incluso habiendo reconocido poco antes que algunas de ellas habían sido perjudiciales para su evolución escolar. Se trata de una desresponsabilización de los y las agentes institucionales externos por toda una serie de discursos y prácticas normalizadas (Horcas et al., 2015) que tienden a hacer que, incluso observando hechos externos que les afectan, los y las estudiantes terminen culpabilizándose y no siendo capaces de buscar razones de su éxito o fracaso más allá de su individualidad.

Sí reconocen de manera generalizada, sin embargo, el influjo del mercado laboral a la hora de transitar por el sistema educativo, aunque siempre lo hacen supeditando esto bien a sus gustos, bien a su capacidad. Lo mismo ocurre con las familias, cuya importancia en lo que se refiere a opinión y apoyo es señalada en la mayoría de las entrevistas; o del profesorado, cuya presencia, si bien es muy inferior a la de las familias, también aparece en algunos casos señalada como relevante en la trayectoria. No obstante, y a pesar de las múltiples influencias que reconocen haber tenido en sus trayectorias, al final solo se ven exclusivamente a sí mismos como únicos responsables de su éxito o fracaso a través de ellas. Tal y como muestran Goodman et al. (2012), la total responsabilización de los y las jóvenes en lo que se refiere a sus resultados y trayectorias, conlleva en los casos de fracaso una pérdida de autoestima que puede fácilmente acabar en abandono del sistema y en autonegación de las propias posibilidades para mejorar e incluso, en el futuro, para encontrar trabajo. De hecho, el autoconcepto se ha revelado como factor clave a la hora de evitar el abandono escolar temprano de los y las jóvenes (García Gracia y Sánchez Gelabert, 2020). Es claramente injusto que lo tengan dañado por la influencia de factores sociales que no dependen de ellos. No obstante, en mayor medida lo es por el influjo de su sensación de incapacidad para interpretar con mayor objetividad sus propias experiencias. Dichas experiencias, como hemos podido observar, no tienen que ver exclusivamente, a diferencia de lo que ellos tienden a pensar, ni con su voluntad ni con su capacidad intelectual. Nos encontramos, por tanto, con casos de injusticia hermenéutica normalizados de carácter tanto social como institucional y que, si bien tienen un reflejo y unas consecuencias más negativas en los colectivos socialmente más vulnerables, afectan a todos los y las jóvenes entrevistados (Medina, 2011).

\section{Conclusiones}

Este artículo tenía como finalidad analizar a qué factores, de carácter estructural o individual, otorgan reconocimiento los y las jóvenes que han transitado de educación obligatoria a educación postobligatoria, a través del relato que hacen tanto de sus trayectorias educativas como de las transiciones y elecciones que han llevado a cabo durante las mismas. Para realizar el análisis partíamos de la noción de injusticia hermenéutica, con el objetivo de identificar en los discursos de los jóvenes su grado de consciencia y, por tanto, capacidad de interpretación holística de todos los factores, estructurales y coyunturales, ajenos y personales, que influyen o condicionan sus elecciones. Los resultados del análisis permiten extraer tres conclusiones.

Los y las jóvenes que estudian el primer curso de Bachillerato y de FPGM muestran diferencias con relación a la uniformidad/discontinuidad de sus respectivas trayectorias, de forma que mientras que las de los estudiantes de Bachillerato son fundamentalmente uniformes, las de los estudiantes de FP presentan una mayor variabilidad y diversidad. Estos resultados permiten identificar y contrastar el influjo de factores estructurales en las trayectorias escolares de los y las jóvenes, y confirman la asociación de la rama de educación postobligatoria con el rendimiento académico, orientándose hacia Bachillerato aquellos jóve- 
nes con un rendimiento académico más alto, y hacia FP los estudiantes cuyos resultados son más bajos.

A pesar de que el estudio de las trayectorias refleja el peso de factores estructurales (sociales, económicos e institucionales), el relato del alumnado se centra en la dimensión individual a la hora de establecer relaciones entre las experiencias y las aspiraciones escolares, tanto de ellos y ellas mismos, como de los y las jóvenes en general, situando el peso de las elecciones en los deseos e intereses del estudiantado en sus capacidades. Dicha interpretación individualista implica una sobre-responsabilización de su trayectoria, la cual, en algunos casos, termina por afectar a su autoconcepto.

Finalmente, y estrechamente relacionado con el aspecto anterior, junto con la atribución explícita de la responsabilidad al individuo, los estudiantes de Bachillerato y CFPGM niegan la influencia de factores externos, especialmente aquéllos relacionados con el género, el nivel socioeconómico y las prácticas institucionales, tres de los principales factores que la literatura ha identificado como determinantes en las trayectorias y las transiciones escolares. Esta negación contrasta con la influencia de factores externos identificada en el análisis de las trayectorias, y confirma, por tanto, la existencia de carencias en los y las jóvenes de recursos para realizar una interpretación ajustada a la realidad, añadiéndose la injusticia hermenéutica a la fehaciente desigualdad de oportunidades materiales.

\section{Referencias bibliográficas}

Alheit, Peter (2015). "The Concept of "biographicity" as background theory of Lifelong learning?". Conferencia presentada en el $4^{\text {th }}$ International Conference Culture, Biography and Lifelong Learning, Pusan National University, 19-21 de marzo de 2015 (en línea). http://cejsh.icm.edu.pl/cejsh/element/ bwmeta1.element.desklight-7bd3d37b-f76e-495b-b98c-f3c1626a50ea/c/01_Alheit.pdf, consultado el 15 de julio de 2020.

Azorín, Cecilia (2019): "Las transiciones educativas y su influencia en el alumnado". Edetania, 55, 223248. DOI: https://doi.org/10.46583/edetania_2019.55.444.

Bernstein, Basil (1975). Class, Codes and Control: Towards a Theory of Educational Transmissions. Londres: Routledge.

Biggart, Andrew y Walther, Andreas (2005): “Coping with Yo-Yo Transitions: Young Adults' Struggle for Support, between Family and State in Comparative Perspective" en Carmen Leccardi y Elisabetta Ruspini (eds.): A New Youth?: Young People, Generations and Family Life. Farnham: Ashgate Publishing.

Boudon, Raymond (1995). Le juste et le vrai: Études sur l'objectivité des valeurs et de la connaissance. París: Fayard.

Bourdieu, Pierre (2007). El sentido práctico. Buenos Aires: Siglo XXI.

Bourdieu, Pierre y Passeron, Jean Claude (1979). The Inheritors: The French Students and Their Relation to Culture. Chicago: The University of Chicago Press.

Brunila, Kritiina; Kurki, Tuuli; Lahelma, Elina; Lehtonen, Jukka; Mietola, Reetta y Palmu, Talma (2011): “Multiple Transitions: Educational Policies and Young People's Post-Compulsory Choices". Scandinavian Journal of Educational Research, 55 (3), 307-324. DOI: https://doi.org/10.1080/00313831.2011.57 6880 
Casal, Joaquim; García, Maribel; Merino, Rafael y Quesada, Miguel (2006): “Aportaciones teóricas y metodológicas a la sociología de la juventud desde la perspectiva de la transición”. Papers, 79, 21-48. DOI: http://dx.doi.org/10.5565/rev/papers/v79n0.798.

Cuconato, Morena y Walther, Andreas (2015): “"Doing Transitions' in Education”. International Journal of Qualitative Studies in Education, 28 (3), 283-296. DOI: https://doi.org/10.1080/09518398.2014.987851.

Decreto 11/2019, de 5 de marzo, del Consejo de Gobierno, por el que se modifica el Decreto 29/2013, de 11 de abril, de Libertad de Elección de Centro Escolar en la Comunidad de Madrid (BOCM núm. 59 de 11 de marzo de 2019).

Elias, Marina y Daza, Lidia (2019): “Configuración y reconfiguración de las expectativas educativas después de la educación obligatoria: un análisis longitudinal”. RISE - International Journal of Sociology of Education, 8 (3), 206-235. DOI: http://dx.doi.org/10.17583/rise.2019.4479.

Fricker, Miranda (2017). Injusticia epistémica. Barcelona: Herder.

Furlong, Andy; Woodman, Dan y Wyn, Johanna (2011): “Changing Times, Changing Perspectives: Reconciling 'Transition' and 'Cultural' Perspectives on Youth and Young Adulthood”. Journal of Sociology, 47 (4), 355-370. DOI: https://doi.org/10.1177\%2F1440783311420787.

Gale, Trevor y Parker, Stephen (2018): "Student Aspiration and Transition as Capabilities for Navigating Education Systems" en Aina Tarabini y Nicola Ingram (eds.): Educational Choices, Transitions and Aspirations in Europe: Systemic, Institutional and Subjective Challenges. Nueva York: Routledge.

Gambetta, Diego (1987). Were They Pushed Or Did They Jump?: Individual Decision Mechanisms in Education. Cambridge: Cambridge University Press.

García Gracia, Maribel y Sánchez Gelabert, Albert (2020): "La heterogeneidad del abandono educativo en las transiciones posobligatorias. Itinerarios y subjetividad de la experiencia escolar”. Papers, 105 (2), 235-257. DOI: http://dx.doi.org/10.5565/rev/papers.2775.

González, Teresa y Bernárdez-Gómez, Abraham (2019): "Elementos y aspectos del centro escolar y su relación con la desafección de los estudiantes". Revista de Investigación en Educación, 17 (1), 5-19.

Goodman, Roger; Imoto, Yuki y Toivonen, Tukka (2012). A Sociology of Japanese Youth: From Returnees to NEETs. Nueva York: Routledge.

Hodkinson, Phil (2009): "Understanding Career Decisionmaking and Progression: Careership Revisited”. Career Research \& Development, 21, 4-17.

Hodkinson, Phil y Sparkes, Andrew C. (1997): “Careership: A Sociological Theory of Decision Making”. British Journal of Sociology of Education, 18 (1), 29-44. DOI: https://doi.org/10.1080/0142569970180102.

Horcas, Vicent y Giménez, Elena (2017): “¿Estudias o trabajas? La toma de decisiones en los itinerarios formativos de jóvenes”. Profesorado, 21 (4), 139-157.

Horcas, Vicent; Bernard, Joan Carles y Martínez, Ignacio (2015): “¿Sueña la juventud vulnerable con trabajos precarios? la toma de decisionesen los itinerarios de (in/ex)clusión educativa”. Profesorado, 19 (3), 210-225. 
Jacovkis, Judith; Montes, Alejandro y Manzano, Martí (2020): “Imaginando futuros distintos. Los efectos de la desigualdad sobre las transiciones hacia la educación secundaria posobligatoria en la ciudad de Barcelona”. Papers, 105 (2), 279-302. DOI: http://dx.doi.org/10.5565/rev/papers.2773.

Kantasalmi, Kari y Holm, Gunilla (2017): "Introducing the Complexity of Educational Diversification" en Kari Kantasalmi y Gunilla Holm (eds.): The State, Schooling and Identity: Diversifying Education in Europe. Londres: Palgrave Macmillan.

Ley Orgánica 8/2013, de 9 de diciembre, para la mejora de la calidad educativa. (BOE núm. 295 de 10 de diciembre de 2013).

McCollum, James (2012): "Hermeneutical Injustice and the Social Sciences: Development Policy and Positional Objectivity". Social Epistemology, 26 (2), 189-200. DOI: https://doi.org/10.1080/02691728. 2011.652212.

McDonald, Kevin (1999). Struggles for Subjectivity: Identity, Action and Youth Experience. Cambridge: Cambridge University Press.

Medina, José (2011): "Hermeneutical Injustice and Polyphonic Contextualism: Social Silences and Shared Hermeneutical Responsibilities”. Social Epistemology, 26(2), 201-220. DOI: https://doi.org/10.108 0/02691728.2011.652214

Murillo, F. Javier y Martínez-Garrido, Cynthia (2018): "Magnitud de la segregación escolar por nivel socioeconómico en España y sus Comunidades Autónomas y comparación con los países de la Unión Europea". RASE. Revista de Sociología de la Educación, 11 (1), 37-58. DOI: https://doi.org/10.7203/ RASE.11.1.10129.

Orden 2509/2013, de 1 de agosto, de la Consejería de Educación, Juventud y Deporte, por la que se regula el procedimiento de admisión de alumnos en centros sostenidos con fondos públicos para cursar Formación Profesional de grado medio. Área de Coordinación Legislativa y Relaciones Institucionales. Subdirección General de Régimen Jurídico y Desarrollo Normativo. S.G.T de la Consejería de Presidencia, Justicia y Portavocía del Gobierno (en línea). http://www.madrid.org/wleg_pub/secure/ normativas/contenidoNormativa.jsf ?opcion=VerPdf\&idnorma=9381\#no-back-button, consultado el 15 de julio de 2020

Petriwskyj, Anne (2014): "Critical Theory and Inclusive Transitions to School” en Bob Perry, Sue Dockett y Anne Petriwskyj (eds.): Transitions to School - International Research, Policy and Practice. Nueva York: Springer.

Prieto, Miriam y Rujas, Javier (2020): “Transiciones a la educación posobligatoria en Madrid: el peso de los factores políticos e institucionales". Papers, 105(2), 183-209. DOI: http:/ /dx.doi.org/10.5565/rev/ papers. 2777.

Prieto, Miriam y Villamor, Patricia (2018): "El impacto de una reforma. Limitación de la autonomía, estrechamiento de la libertad y erosión de la participación". Archivos Analíticos de Políticas Educativas, 26 (63), 1-36. DOI: https://doi.org/10.14507/epaa.26.3255. 
Reay, Diana (2010): "Identity-Making in Schools and Classrooms" en Margaret Wetherall y Chandra Talpade Mohanty (eds.): The SAGE Handbook of Identities. Los Angeles: Sage Publications.

Real Decreto-ley 5/2016, de 9 de diciembre, de medidas urgentes para la ampliación del calendario de implantación de la Ley Orgánica 8/2013, de 9 de diciembre, para la mejora de la calidad educativa (BOE núm. 298 de 10 de diciembre de 2016).

Saura, Geo y Luengo, Julián (2015): “Biopolítica y educación. Medición, estandarización, regularización poblacional”. Teoría de la Educación, 27 (2), 115-135. DOI: http://dx.doi.org/10.14201/teoredu2015272115135

Tarabini, Aina y Curran, Marta (2019): 'Young People's Educational Expectations, Aspirations and Choices: The Role of Habitus, Gender and Field" en Garth Stahl, Derron Wallace, Ciaran Burke y Steven Threadgold (eds.): International Perspectives on Theorizing Aspirations: Applying Bourdieu's Tools. Londres: Bloomsbury Academic.

Tarabini, Aina y Jacovkis, Judith (2019): “Transicions a l'educació secundària postobligatòria a Catalunya" en Jordi Riera (dir.): Reptes de l'educació a Catalunya. Anuari 2018. Barcelona: Fundació Jaume Bofill.

Tarabini, Aina e Ingram, Nicola (2018): “Introduction” en Aina Tarabini y Nicola Ingram (eds.): Educational Choices, Transitions and Aspirations in Europe: Systemic, Institutional and Subjective Challenges. Nueva York: Routledge.

Tarabini, Aina; Curran, Marta y Fontdevila, Clara (2015): "El habitus institucional: una herramienta teórica y metodológica para el estudio de la cultura escolar". Témpora, 18, 37-58.

Walther, Andreas; Warth, Annegret; Ule, Mirjana y Du Bois-Reymond, Manuela (2015): “"Me, my Education and I': Constellations of Decision-Making in Young People's Educational Trajectories". International Journal of Qualitative Studies in Education, 28 (3), 349-371. DOI: https://doi.org/10.1080/0951 8398.2014.987850.

Walther, Andreas (2006): "Regimes of Youth Transitions. Choice, Flexibility and Security in Young People's Experiences Across Different European Contexts". Young: Nordic Journal of Youth Research, 14 (2), 119-139. DOI: https://doi.org/10.1177\%2F1103308806062737.

\section{Notas biográficas}

Alberto Sánchez Rojo es Doctor Europeo con Premio Extraordinario en Conocimiento Pedagógico Avanzado (2016) por la Universidad Complutense de Madrid, donde ejerce actualmente como Profesor Ayudante Doctor y Secretario Académico en el Departamento de Estudios Educativos. Miembro activo del Grupo de Investigación en Antropología y Filosofía de la Educación (GIAFE) y del Grupo de Investigación en Procesos, Espacios y Prácticas Educativas (GIPEP), ha ejercido docencia en varias universidades tanto públicas como privadas, así como ha publicado artículos, capítulos de libro y realizado ponencias en ámbitos nacionales e internacionales. Sus líneas de investigación se centran fundamentalmente en el análisis de la realidad educativa desde una perspectiva teórica, social y política. 
Miriam Prieto Egido es Doctora en Pedagogía por la Universidad Complutense de Madrid (2012), Profesora Ayudante Doctora del Departamento de Pedagogía de la Universidad Autónoma de Madrid y miembro del Grupo de Investigación sobre Políticas Educativas Supranacionales (GIPES) de la UAM. Su investigación gira en torno al impacto de los discursos y políticas educativos en el concepto de educación y la práctica de la escolarización. Ha participado en varios proyectos de investigación dirigidos al análisis de políticas educativas a nivel internacional, nacional y regional, con especial foco en las políticas educativas de la Comunidad de Madrid, dirigiendo sus análisis hacia los efectos de medidas como la libertad de elección de centro, la rendición de cuentas o la autonomía escolar. 